\title{
Astronaut-Induced Disturbances to the Microgravity Environment of the Mir Space Station
}

\author{
Dava J. Newman* and Amir R. Amir ${ }^{\dagger}$ \\ Massachusetts Institute of Technology, Cambridge, Massachusetts 02139 \\ and \\ Sherwin M. Beck \\ NASA Langley Research Center, Hampton, Virginia 23681
}

\begin{abstract}
In preparation for the International Space Station, the Enhanced Dynamic Load Sensors Space Flight Experiment measured the forces and moments astronauts exerted on the Mir Space Station during their daily on-orbit activities to quantify the astronaut-induced disturbances to the microgravity environment during a long-duration space mission. An examination of video recordings of the astronauts moving in the modules and using the instrumented crew restraint and mobility load sensors led to the identification of several typical astronaut motions and the quantification of the associated forces and moments exerted on the spacecraft. For 2806 disturbances recorded by the foot restraints and hand-hold sensor, the highest force magnitude was $137 \mathrm{~N}$. For about $96 \%$ of the time, the maximum force magnitude was below $60 \mathrm{~N}$, and for about $99 \%$ of the time the maximum force magnitude was below $90 \mathrm{~N}$. For $95 \%$ of the astronaut motions, the rms force level was below $9.0 \mathrm{~N}$. It can be concluded that expected astronaut-induced loads from usual intravehicular activity are considerably less than previously thought and will not significantly disturb the microgravity environment.
\end{abstract}

$F_{\text {mag }}$
$F_{\text {mag,max }}$
$F_{\text {rms }}$
$F_{x}, F_{y}, F_{z}$
$f_{k}$
$f_{\text {sampling }}$
$I_{r}\left[f_{k}\right]$
$k$
$L$
$M_{\text {mag }}$
$M_{\text {mag,max }}$
$M_{\text {rms }}$
$N_{1}$
$N_{2}$
$n$
$p$
$r$
$S_{x}\left[f_{k}\right]$
$U$
$w[n]$
$X_{r}[k]$
$x[n]$
$x_{r}[n]$

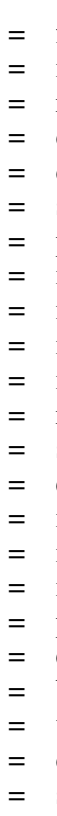

\section{Nomenclature}

force magnitude

maximum force magnitude

rms force

discrete force components

discrete Fourier transform frequency, $k / L$

sampling frequency

periodogram

index variable

number of samples

moment magnitude

maximum moment magnitude

rms moment

start index of the event

end index of the event

index variable

momentum

index variable ranging from 1 to $R$

power spectral density estimate

energy in the window

boxcar window function

windowed fast Fourier transform

discrete signal

$=$ subsequences of signal $x[n]$

\section{Introduction}

T HE first phase of the International Space Station (ISS) Program, known as the Shuttle-Mir Program, provided the United States the opportunity to send astronauts to the Russian space station Mir and conduct long-duration space flight experiments. The primary objective was to reduce the technical risk associated with the construction and operation of the ISS. Previous space stations and the shuttle have been used quite extensively for microgravity research, but the ISS is the first human-occupied space vehicle

Received 10 April 2000; revision received 17 June 2000; accepted for publication 23 January 2001 . Copyright (C) 2001 by the authors. Published by the American Institute of Aeronautics and Astronautics, Inc., with permission.

${ }^{*}$ Associate Professor of Aeronautics and Astronautics. Senior member AIAA.

${ }^{\dagger}$ Research Engineer, Man-Vehicle Laboratory, Department of Aeronautics and Astronautics. Senior Member AIAA.

${ }^{\ddagger}$ Senior Technical Manager. developed with system requirements specifying the microgravity environment. ISS requirements call for microgravity operations for 30-day durations where disturbing accelerations and vibrations are reduced and, if possible, eliminated. ${ }^{1,2}$ Within the framework of the Shuttle-Mir Program, the Enhanced Dynamic Load Sensors (EDLS) experiment was conducted on Mir to quantify the disturbances to the microgravity environment caused by the presence of astronauts.

In designing the ISS, NASA and its contractors adopted maximum force levels recorded during the T-013 experiment on the Skylab orbital station in August of 1973 as the expected disturbance loads from astronaut motion. In this experiment an astronaut executed a set of prescribed activities (console operation, flapping arms, bowing, respiration exercises, etc.) while the reaction forces and moments were measured. The activities included "vigorous soaring" as a worst-case scenario, resulting in maximum loads close to $500 \mathrm{~N}$ and a maximum soaring velocity of approximately $1.9 \mathrm{~m} / \mathrm{s}$ (Refs. 3-6). Exclusive reliance on the limited Skylab database was considered questionable for several reasons. The prescribed activities are not representative of routine astronaut motions, and maximum loads arose from vigorous soaring activities that were meant to measure a worst-case scenario. Furthermore, almost the entire data set was recorded in a single session lasting less than $80 \mathrm{~min}$ and stemming from a single astronaut subject. Two short additional sessions were conducted to address anomalies found after the first run. While a second astronaut participated in the soaring activities, data were recorded for only the primary subject. ${ }^{3,4}$

As a result, a more comprehensive measurement of astronaut intravehicular activity (IVA) loads was conducted on board the space shuttle during mission STS-62 (4-18 March 1994) under the Dynamic Load Sensors (DLS) investigation. The purpose of DLS was to quantify the forces and moments exerted by the astronauts on the orbiter middeck as they are going about their daily IVA. ${ }^{7,8}$ The key hardware component of DLS was a set of three sensors: a touchpad, a foot restraint, and a handhold, similar in appearance to the restraint devices found in the orbiter that assist the crew in moving about the vehicle. ${ }^{9}$ Data were recorded over a period of $67 \mathrm{~h}$ during flight days 7, 8, and 11 of the 14-day mission. Altogether 301 astronaut motions were found through a correlation with video footage recorded inside the middeck.

The EDLS experiment was proposed as a follow-on to DLS to expand the shuttle database by collecting astronaut reaction forces and moments on Russia's Mir space station. The limited volume of the space shuttle middeck coupled with the constant activity of 
numerous crewmembers limits the applicability of the data to IVA operations during a long-duration space flight. In addition, shuttle missions normally last about two weeks, and as a result there is insufficient time to observe long-term astronaut adaptation to weightlessness. A general-purpose acceleration measurement system, Space Acceleration Measurement System (SAMS),${ }^{10,11}$ flew on Mir, which provided the opportunity to correlate the astronaut-induced forces and moments with the accelerations experienced by a Mir module or the entire complex ${ }^{12-14}$ and establish transfer functions. ${ }^{15}$

Historically, early investigations of astronaut-spacecraft interaction focused on how the spacecraft attitude would be perturbed by the astronauts and how to account for this disturbance source in the design of the vehicle attitude control system. This issue was first raised in $1962 .{ }^{16}$ The question of astronaut motion potentially disturbing the microgravity environment specified for scientific experiments became a primary concern with the beginning of the space shuttle program and the launch of the Russian space station Mir in the 1980 s. $^{8}$

The low-frequency acceleration level experienced by a spacecraft in low Earth orbit is approximately $10^{-6} \mathrm{~g}$. A level of $10^{-7} \mathrm{~g}$ can be achieved over a very small region near the center of mass. The accelerations experienced by the spacecraft are classified fundamentally as quasi-steady, oscillatory, or transient. ${ }^{17}$

Quasi-steady accelerations below $0.01 \mathrm{~Hz}$ are produced by external forces and kinematics. ${ }^{1}$ Oscillatory accelerations (so-called "g-jitter") are generated within the spacecraft in the region 0.01 to $300 \mathrm{~Hz}$. Sources of oscillatory disturbances are the mechanical motions of reciprocating pumps, fans, motors, gyros, and other devices, as well as the acoustic noise from fans, duct inlets/outlets, pumps, blowers, etc. Astronaut exercise activity on a treadmill or ergometer can also produce a strong periodic perturbation to the microgravity environment. Reaction forces and moments caused by astronaut motion are transient accelerations (i.e., spikes) and have typically a duration of less than a second and are nonperiodic. The energy in the disturbance is usually spread across a broad frequency range. The structural modes of the spacecraft are often excited by transient accelerations, and thus the dynamic response can last much longer than the actual perturbation. ${ }^{17}$

The sensitivity to the acceleration level and frequency varies greatly by type of experiment conducted in orbit. Although an overall acceleration level of less than $10^{-3} \mathrm{~g}$ is sufficient for some experiments, $10^{-8} \mathrm{~g}$ may be specified for others. To put the magnitude of disturbances produced by the astronauts into perspective, the Space Shuttle Orbiter's acceleratory environment shifts from microgravity to as much as $10^{-4} \mathrm{~g}$ during the firing of the vernier thrusters used for fine attitude control, resulting in a $10^{-2} \mathrm{~g}$ environment. ${ }^{17}$ [The Space Shuttle Orbiter is equipped with six vernier thrusters (2 forward and $4 \mathrm{aft}$ ), which are used for fine attitude control. Each thruster is rated at $107 \mathrm{~N}(24 \mathrm{lb})$ of thrust.]

\section{Methods}

The EDLS experiment measured daily astronaut IVA activities and prescribed motions. In a daily IVA session data acquisition was activated, and the astronauts went about their scientific or operational activities. Whenever the EDLS sensors detected loads exceeding a specified minimum threshold level, data were recorded on the storage medium. One such recording is known as an event, which is understood as either a specific astronaut motion lasting from a few seconds to several minutes or several motions together without an interruption in the loading to a sensor. If a distinction of individual motions in the latter case is needed, they are referred to as subevents. In prescribed motion sessions the astronaut subjects used one or more foot restraint sensors while throwing a small ball of approximately $2 \mathrm{~cm}$ in diameter at a target $1.5-2 \mathrm{~m}$ away for repeated trials with their eyes either open or closed. The purpose of the throwing activity was to quantify the adaptation of human motor control in microgravity, and results are published elsewhere. ${ }^{18}$

EDLS data recording began during the NASA 2/Mir 21 mission and between 24 May and 30 August 1996; 33 EDLS data recording sessions were conducted of which 31 recorded daily activity and two recorded throwing activity of the three crew members. Because of an on-orbit failure of the data acquisition computer, ${ }^{19}$ it was not
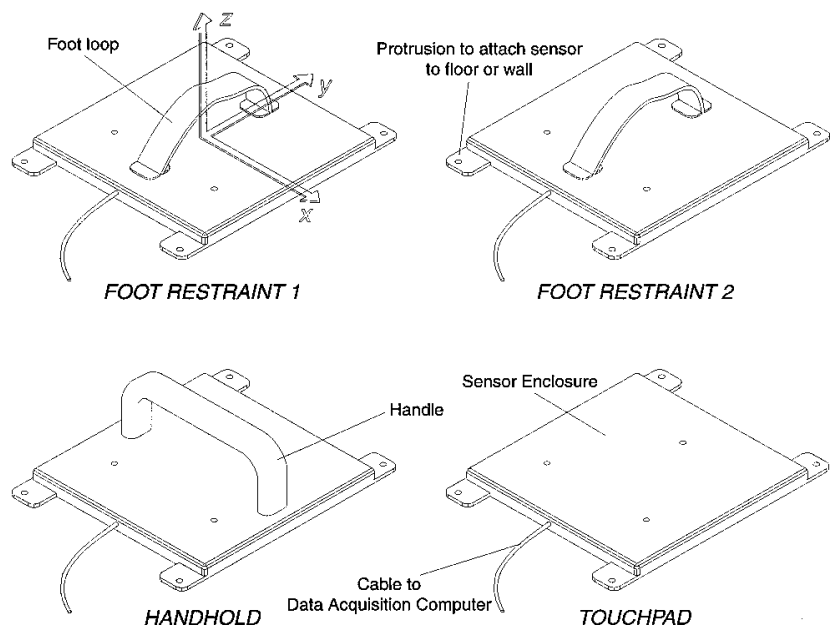

Fig. 1 Four EDLS sensors used onboard the Russian space station $\mathrm{Mir}$ to measure the reaction forces and moments produced by the astronauts.

possible to record EDLS data during the NASA 3/Mir 22 mission. A similar computer system on Mir for another experiment ${ }^{20}$ was modified to act as a substitute data acquisition system with EDLS sessions resuming during the NASA 4/Mir 22/Mir 23 mission from 25 February to 9 May 1997. During this mission, 17 total sessions were conducted including 11 daily IVA activity sessions and six throwing sessions in which all Mir crew members participated. During each EDLS session, numerous crew members exerted IVA loads through the sensors. Following the collision of a Progress resupply vehicle with the Spektr module of Mir in June 1997 and the resulting difficulties on the station, astronauts were not available to perform additional EDLS sessions. The overall data recording time was $133 \mathrm{~h}$ during the 13 months EDLS was operating on Mir.

The key hardware component of the experiment was a set of four six-degree-of-freedom load sensors consisting of an instrumented handhold, touchpad, and two foot restraints. The sensors, shown in Fig. 1, were designed to provide the same functionality as the foot loops and hand rails built into the Space Shuttle Orbiter and the Mir orbital complex for astronaut use. The sensors are interchangeable. The touchpad's functionality was envisioned to be that of a flat surface the astronauts would use to push themselves off using either their hands or feet.

Each sensor, with dimensions of $24 \times 24 \times 2 \mathrm{~cm}$ (without attachments), measures forces and torques with three custom-designed load cells (so-called flexures) in a circular arrangement placed $120 \mathrm{deg}$ apart. Each load cell is equipped with two full Wheatstone strain gauge bridges consisting of four foil strain gauges. The sensor feeds the data acquisition computer with six signals that measure the deflection of the load cells. However, only three of the six signals from the touchpad sensor were actually sampled because of the limited number of channels on the A/D converter, so that only translational load information is available for that unit. The sensor enclosure and the flexures were designed for a microgravity full scale load of $400 \mathrm{~N}$ of force and $50 \mathrm{~N} \cdot \mathrm{m}$ of torque. ${ }^{9}$ The unit housing the experiment computer, the signal conditioning system, the storage mechanism, and related components, is referred to as the Experiment Support Module (ESM). Because only 15 data acquisition channels were available, at any given time at most three sensors could be used resulting in two possible sensor configurations: 1) two foot restraint sensors and a touchpad sensor; and 2) a foot restraint sensor, a handhold sensor, and a touchpad sensor. In the case of the original ESM (used during NASA 2/Mir 21), sensor signals were sampled with a 13-bit A/D converter at a rate of $250 \mathrm{~Hz}$. To avoid aliasing of the data, the signals were passed through an eighth-order low-pass Bessel filter with a corner frequency of $125 \mathrm{~Hz}$. For the substitute ESM (used during NASA 4/Mir 22/Mir 23) A/D conversion was performed with 16-bit resolution and filtered with a fourth-order Bessel filter. The filter corner frequency and the sampling rate were the same for both systems.

To convert the load cell deflection signals measured by a sensor into an applied load, a $6 \times 6$ calibration matrix is required, which 
converts the six measured voltages into three applied force and three applied moment components reflecting various properties of a sensor's specific enclosure, load cells, strain gauges, etc. ${ }^{9}$ Following the 24 months on Mir, the sensors were recalibrated postflight with the substitute ESM once the hardware returned to the ground laboratory. The accuracy was within $\pm 1.6 \%$ of the full-scale load for all four sensors. ${ }^{19}$ Transformation into forces and moments was made with the preflight calibration matrix for the data recorded during the NASA 2/Mir 21 mission (with the original ESM) and the postflight calibration matrix for the data recorded during the NASA 4/Mir 22/Mir 23 mission (with the substitute ESM). All data were low-pass filtered with a digital three-pole elliptical filter with a cutoff frequency of $30 \mathrm{~Hz}$.

\section{Analysis}

From the entire data set collected, individual events (i.e., individual astronaut motions) were identified. The force traces for a typical event recorded by the handhold sensor are shown in Fig. 2. In this example the event lasts from time index $0.4 \mathrm{~s}$ to time index $3.8 \mathrm{~s}$. Each event was verified manually. Although this approach was time con- suming, it ensured high-fidelity data analysis without the omission of "interesting" but valid data. Overall, 4819 events were identified with the breakdown by mission and sensor type provided in Table 1. These events are contained in 1257 data files and represent a total contact time of the astronauts with the sensors of $19.3 \mathrm{~h}$. The data from the touchpad sensor are not considered further because it was used (unintended) as a foot restraint in a location underneath a standard Mir hand rail affixed to the floor. Forces were recorded from the EDLS touchpad, but no measure of the load applied to the Mir hand rail were possible in this configuration. The EDLS handhold

Table 1 Number of astronaut motions recorded

\begin{tabular}{lccr}
\hline \hline Type of sensor & NASA 2 mission & NASA 4 mission & Total \\
\hline Foot restraint sensor 1 & 1817 & 326 & 2143 \\
Foot restraint sensor 2 & 362 & 0 & 362 \\
Handhold sensor & 230 & 71 & 301 \\
Touchpad sensor & 1089 & 585 & 1674 \\
Total & 3498 & 982 & 4480 \\
\hline \hline
\end{tabular}
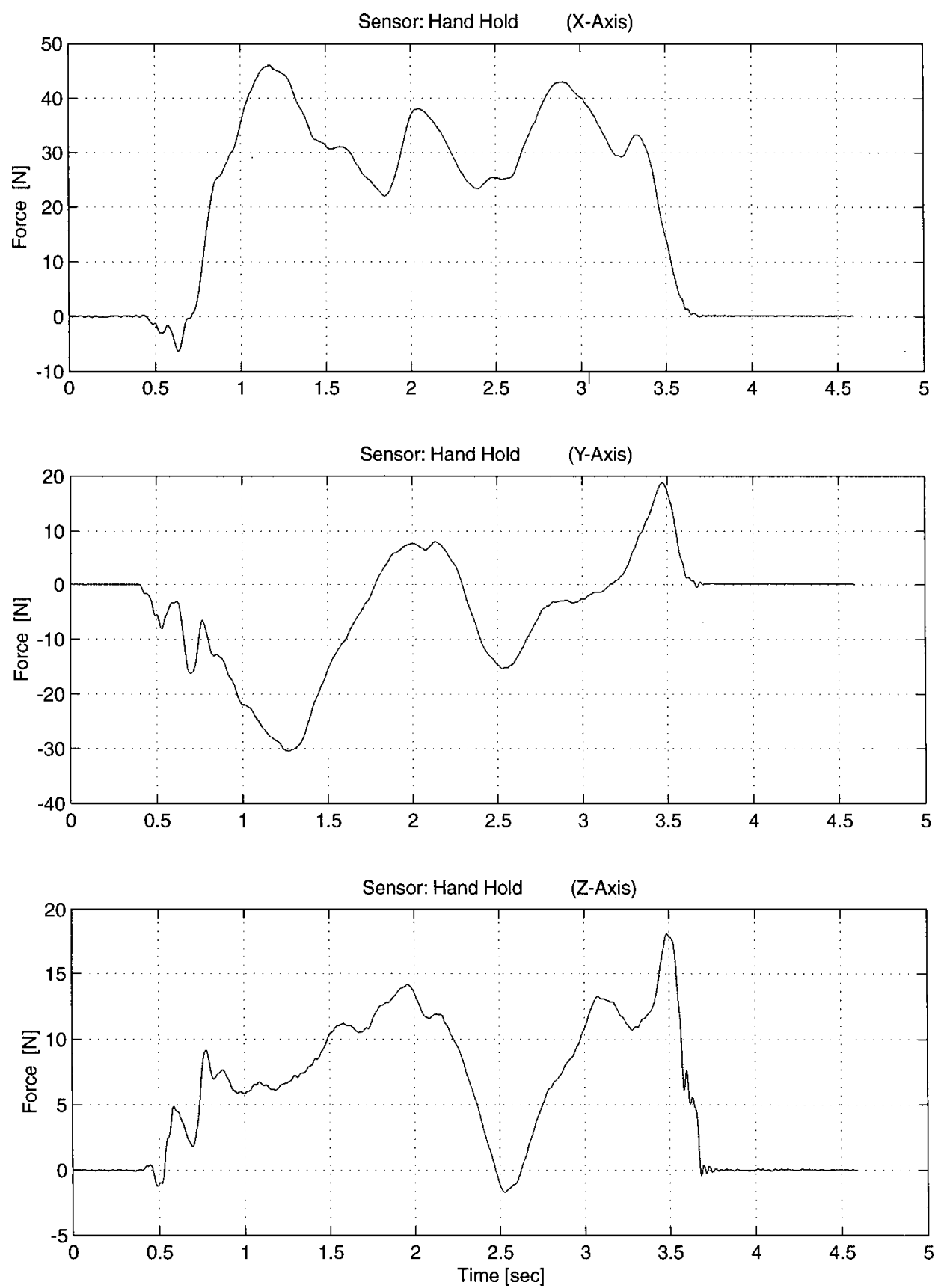

Fig. 2 Three force components for an event recorded by the handhold sensor on 10 July 1996 during the NASA 2/Mir 21 mission to Mir. 
and foot restraints encompass restraint design slated for the ISS, and all data were analyzed.

The analysis was conducted in both the time and the frequency domain. As part of the former, the maximum force magnitude (i.e., the magnitude of the force vector) occurring in each event is computed:

$$
F_{\text {mag, } \max }=\max _{N_{1} \leq n \leq N_{2}}\left(\sqrt{F_{x}^{2}[n]+F_{y}^{2}[n]+F_{z}^{2}[n]}\right)
$$

The rms force is a measure of the average load:

$$
F_{\mathrm{rms}}=\sqrt{\left(\sum_{n=N_{1}}^{N_{2}}\left(F_{x}^{2}[n]+F_{y}^{2}[n]+F_{z}^{2}[n]\right)\right) / N}
$$

Similarly, the maximum moment magnitude $M_{\mathrm{mag}, \max }$ and rms moment $M_{\text {rms }}$ are determined for each event. For some applications the largest force component rather than the force magnitude is of interest, and therefore the largest of three force components is determined and its axis noted. The total momentum transferred in each event is the force magnitude integrated over time, which was approximated with a trapezoidal rule so that the momentum $p$ is determined by the formula

$$
p=\frac{1}{f_{\text {sampling }}} \sum_{n=N_{1}}^{N_{2}} F_{\text {mag }}[n]
$$

In the frequency domain analysis the distribution of power in the astronaut-induced disturbance was computed. The power spectral density (PSD) was used to calculate the frequency below which 95\% (two standard deviations) of the signal's energy in the event was contained. For the discrete signal $x[n]$ recorded by the sensors, the PSD was estimated using Welch's Method of Modified Periodograms. ${ }^{21}$ The signal $x[n]$ is decomposed into subsequences $x_{r}[n]$ of length $L$ samples such that $r$ ranges from 1 to $R$. For each of the subsequences $x_{r}[n]$, the windowed fast Fourier transform $X_{r}[k]$ was computed as follows:

$$
X_{r}[k]=\sum_{n=0}^{L-1} x_{r}[n] w[n] \exp \left(\frac{-j 2 \pi n k}{L}\right)
$$

The periodogram $I_{r}\left[f_{k}\right]$ is computed with the following expression:

$$
I_{r}\left[f_{k}\right]=(1 / U)\left|X_{r}[k]\right|^{2}
$$

where $U$ is calculated as follows:

$$
U=\sum_{n=0}^{L-1}(w[n])^{2}
$$

The PSD estimate $S_{x}\left[f_{x}\right]$ is then the weighted sum of the periodograms of each of the individual subsequences:

$$
S_{x}\left[f_{k}\right]=\frac{1}{R} \sum_{r=1}^{R} I_{r}\left[f_{k}\right]
$$

With the PSD distribution known the trapezoidal method was used to estimate the area underneath the PSD curve and a secondorder interpolation to obtain the frequency below which $95 \%$ of the energy content lies for the frequency analysis.

The EDLS experiment measured the disturbances to the microgravity environment caused by the astronauts for a wide variety of typical astronaut motions. For example, at the low end of the disturbance spectrum is the contact of the astronaut's toes with the sensors during a position/posture adjustment while using the foot restraint sensors. On the other end of the spectrum is a forceful push-off away from the sensors as the astronaut has completed the work and wants to move to another module. The number of occurrences of the first mentioned event is far larger than that of the second. For this reason the mean force/moment/momentum measurement is fairly low, but the standard deviation for all events is large given the wide magnitude range of events measured.

\section{Results}

The characteristic motions observed during EDLS were very similar to those seen during DLS, which have been described. ${ }^{8}$ Figure 3 shows a histogram of the maximum force magnitudes recorded by the two foot restraint sensors and the handhold sensor in the EDLS experiment. For these 2806 events $96 \%$ of the time the maximum force magnitude was below $60 \mathrm{~N}$, and about $99 \%$ of the time the maximum force magnitude was below $90 \mathrm{~N}$. For each force magnitude interval the frequency of occurrence is provided. For example, about $17.4 \%$ of all events have a maximum force magnitude between 20 and $30 \mathrm{~N}$. The highest force magnitude measured by the two foot restraint sensors was $137 \mathrm{~N}$ and for the handhold sensor $124 \mathrm{~N}$. Assuming a total mass of 400 metric tons for the ISS at assembly complete, an applied force of $137 \mathrm{~N}$ would result in an acceleration of about $3.5 \times 10^{-5} \mathrm{~g}$ excluding dynamic effects of the

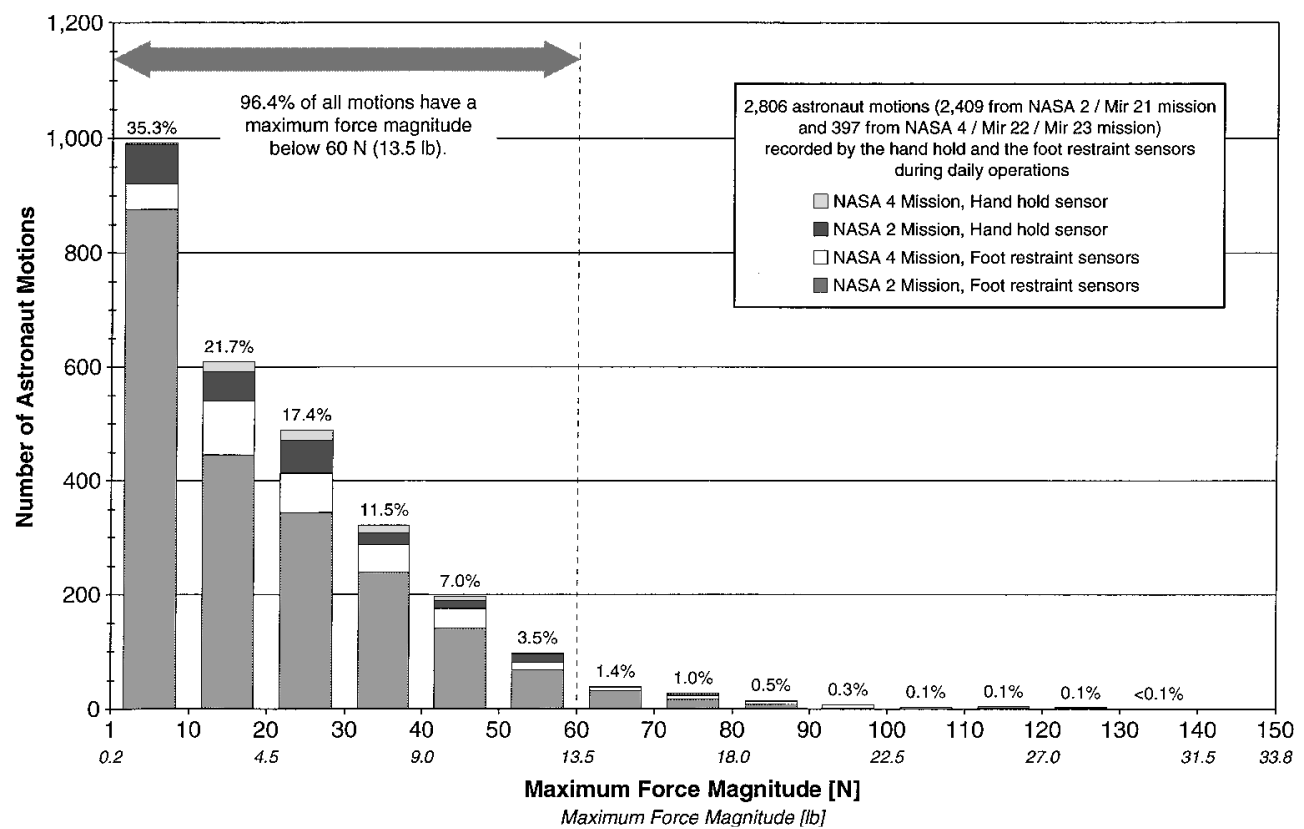

Fig. 3 Histogram of the maximum force magnitudes recorded by the two foot restraint sensors and the handhold sensor. 
structure. The average force magnitude recorded was $21 \pm 18 \mathrm{~N}$, and the median force magnitude was $16 \mathrm{~N}$. The normal or $z$ component of the applied force was the largest of the three components $59 \%$ of the time. The duration of events ranged from 0.1 to $557.2 \mathrm{~s}$ with an average of $15.0 \pm 36.1$ and a median value of 3.7. The distribution of the rms force values for the events is shown in Fig. 4. The largest rms force was $35.6 \mathrm{~N}$, the average value was $2.4 \pm 3.1 \mathrm{~N}$, and the median value $1.3 \mathrm{~N}$. For $96 \%$ of the astronaut motions, the rms force value was below $9.0 \mathrm{~N}$.

A histogram of the maximum moment magnitudes is shown in Fig. 5. The largest moment was measured by foot restraint 1 with a value of $18.3 \mathrm{~N} \cdot \mathrm{m}$. The average moment was $1.9 \pm 2.2 \mathrm{~N} \cdot \mathrm{m}$. The distribution of the moment magnitude for the 2806 events was such that $96 \%$ of all events had a maximum moment magnitude below $7 \mathrm{~N} \cdot \mathrm{m}$. The average momentum imparted by the astronauts on the station was $83.1 \pm 228.2 \mathrm{~kg} \cdot \mathrm{m} / \mathrm{s}$ in the 2806 events. An analysis of the power spectral density distribution in the events showed that on average $95 \%$ of the power was contained below a frequency of $2.6 \pm 2.4 \mathrm{~Hz}$. The results of the statistical analysis of the astronaut motions are summarized in Table 2 .
Table 2 Summary of the results from the statistical analysis of the astronaut motions

\begin{tabular}{|c|c|c|c|}
\hline Measurement & $\begin{array}{l}\text { Foot restraint } \\
1 \text { and } 2 \\
\text { sensors }\end{array}$ & $\begin{array}{l}\text { Handhold } \\
\text { sensor }\end{array}$ & $\begin{array}{l}\text { Foot restraint } 1 \text {, } \\
\text { foot restraint } 2 \text {, } \\
\text { handhold sensors }\end{array}$ \\
\hline \multicolumn{4}{|l|}{ Force magnitude } \\
\hline Maximum value, $\mathrm{N}$ & 136.6 & 124.0 & 136.6 \\
\hline Average value, $\mathrm{N}$ & $20.7 \pm 18.1$ & $25.1 \pm 20.5$ & $21.1 \pm 18.4$ \\
\hline Median value, $\mathrm{N}$ & 15.6 & 21.7 & 16.2 \\
\hline \multicolumn{4}{|l|}{ Moment magnitude } \\
\hline Maximum value, $\mathrm{N} \cdot \mathrm{m}$ & 18.31 & 14.24 & 18.31 \\
\hline Average value, $\mathrm{N} \cdot \mathrm{m}$ & $1.83 \pm 2.10$ & $2.56 \pm 2.47$ & $1.91 \pm 2.15$ \\
\hline Median value, $\mathrm{N} \cdot \mathrm{m}$ & 1.08 & 1.82 & 1.13 \\
\hline \multicolumn{4}{|l|}{ Rms force } \\
\hline Maximum value, $\mathrm{N}$ & 35.6 & 33.5 & 35.6 \\
\hline Average value, $\mathrm{N}$ & $2.3 \pm 2.9$ & $3.8 \pm 4.5$ & $2.4 \pm 3.1$ \\
\hline Median value, $\mathrm{N}$ & 1.2 & 2.7 & 1.3 \\
\hline Average duration, s & 16.3 & 4.9 & 15.0 \\
\hline $\begin{array}{l}\text { Average momentum } \\
\text { transfer, } \mathrm{kg} \cdot \mathrm{m} / \mathrm{s}\end{array}$ & 87.3 & 47.5 & 83.1 \\
\hline
\end{tabular}

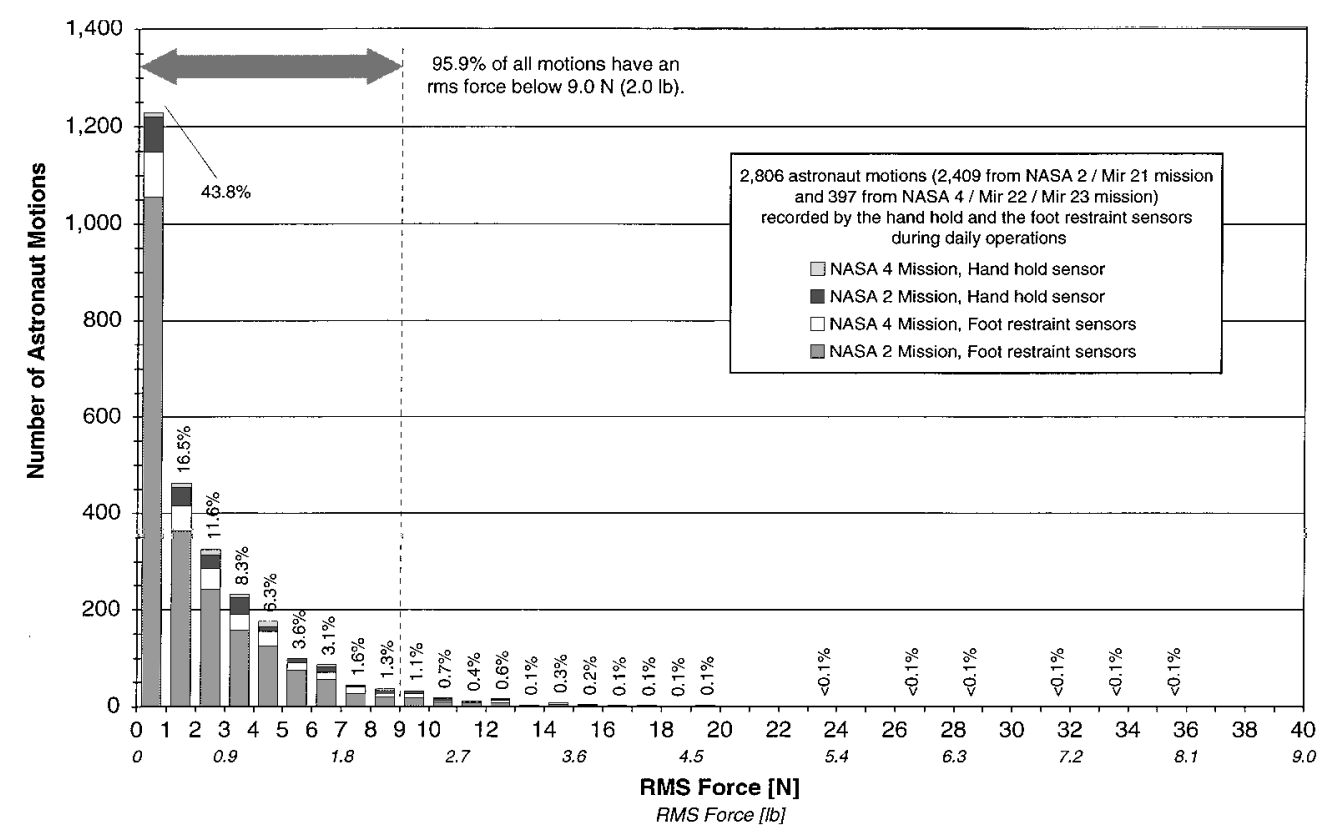

Fig. 4 Histogram of the rms forces recorded by the two foot restraint sensors and the handhold sensor.

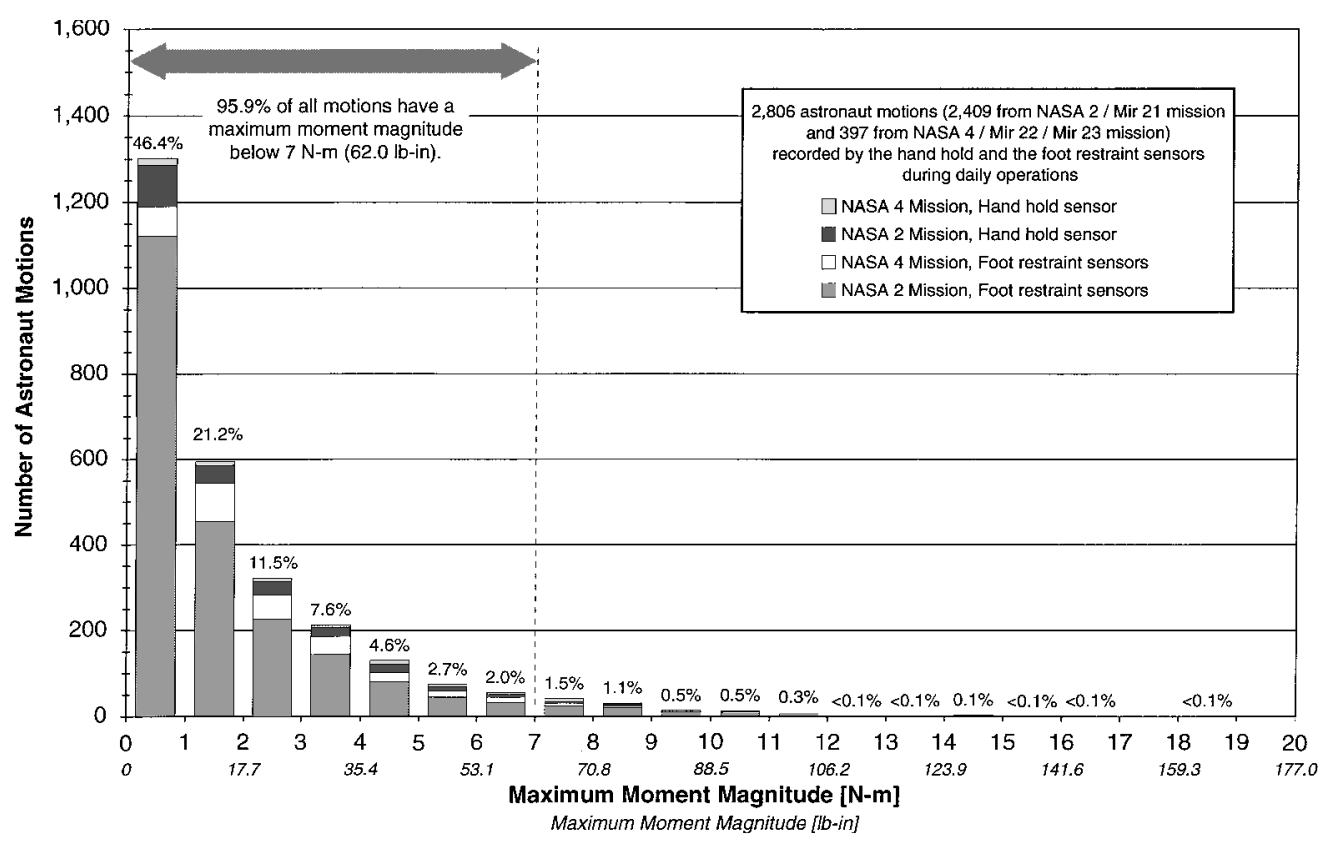

Fig. 5 Histogram of the maximum moment magnitudes recorded by the two foot restraint sensors and the handhold sensor. 
As the astronauts adapt to the microgravity environment in orbit during the first several weeks in space, it is expected that their motions decrease in velocity and magnitude. Because of the high operational demand on the crew during the spacecraft hand-over time and shortly thereafter, the astronauts recorded EDLS sessions after the first three weeks in space. Overall, the EDLS experiment did not observe any significant change in the average force level observed.

\section{Conclusions}

The EDLS experiment produced the first comprehensive description of IVA astronaut motions and quantified astronaut-induced loads in microgravity for long-duration space flight. Living on orbit, astronauts adapt to microgravity resulting in finely controlled motions, slow velocities, and low force levels. Astronaut-induced loads expected on the ISS from usual IVA are considerably smaller than previously thought and will not significantly disturb the ISS microgravity environment.

Current specifications for ISS microgravity operations do not include detailed effects of crew activity, but do include effects caused by usage of crew equipment, such as the operation of exercise devices and latched or hinged enclosures. The specifications state only that "crew effects will be mitigated to the extent possible.". The magnitude of astronaut-induced loads is difficult to infer from visual observation but active sensing with real-time feedback of the applied force and torque levels could be a valuable tool for the astronauts during microgravity operations.

\section{Acknowledgments}

The EDLS space flight experiment was funded by NASA under Contract NAS1-18690. The authors wish to express their appreciation for the support and help provided by Javier de Luis, Kim Scholle, Ed Bokhour, and Joseph Zapetis of Payload Systems, Inc.; Marthi-nus van Schoor of Midé Technology Corporation; Peter Bruckner and Joel Gwynn of Designer's CADD, Inc.; Massachusetts Institute of Technology graduate students Natasha Neogi and Michail Tryfonidis, and undergraduate students Jennifer Bonnell, Alan Chen, Rebeka Marcus, and Larry Pilkington.

\section{References}

1“Microgravity Control Plan,” NASA SSP 50036, Rev. A, Type 2, Feb. 1996.

2"System Specification for the International Space Station," NASA SSP 41000, Rev. H, May 1998.

${ }^{3}$ Conway, B. A., and Hendricks, T. C., "A Summary of the Skylab Crew/Vehicle Disturbance Experiment T-013," NASA TN D-8128, March 1976

${ }^{4}$ Conway, B. A., "Investigation of Crew Motion Disturbances on SkylabExperiment T-013," Advances in the Astronautical Sciences, Vol. 31, Pt. 1, 1975, pp. 445-464.

${ }^{5}$ Kullas, M. C., "Handbook on Astronaut Crew Motion Disturbances for Control System Design,” NASA Rept. RP-1025, NASA Langley Research Center, Hampton, VA, May 1979.

${ }^{6}$ Dunbar, B., Giesecke, R., and Thomas, D., "The Microgravity Environ- ment of the Space Shuttle Columbia Payload Bay During STS-32," NASA TP 3141, Nov. 1991

${ }^{7}$ Newman, D. J., Tryfonidis, M., and van Schoor, M., "Astronaut-Induce d Disturbances in Microgravity," Journal of Spacecraft and Rockets, Vol. 34, No. 2, 1997, pp. 252-254.

${ }^{8}$ Amir, A. R., and Newman, D. J., "Research into the Effects of Astronaut Motion on the Spacecraft: A Review," Acta Astronautica, Vol. 47, No. 12, 2000, pp. 859-869.

${ }^{9}$ Amir, A. R., Baroni, G., Pedrocchi, A., Newman, D., Ferrigno, G., and Pedotti, A., "Measuring Astronaut Performance on the ISS: Advanced Kinematic and Kinetic Instrumentation," Proceeding s of the 16th Inst of Electrical and Electronics Engineers Instrumentation and Measurement Technology Conference, IEEE Publication CH36309, Inst. of Electrical and Electronics Engineers, Piscataway, NJ, 1999, pp. 397-402.

${ }^{10}$ DeLombard, R., and Finley, B. D., "Space Acceleration Measurement System Description and Operation on the First Spacelab Life Sciences Mission,” NAS A TM-105301, Nov. 1991.

${ }^{11}$ DeLombard, R., Finley, B. D., and Baugher, C. R., "Development of and Flight Results from the Space Acceleration Measurement System (SAMS)," NASA TM-105652; also AIAA Paper 92-0354, Jan. 1992.

${ }^{12}$ DeLombard, R., Ryaboukh, S., Hrovat, K., and Moskowitz, M., "Further Analysis of the Microgravity Environment on Mir Space Station During Mir16," NASA TM-107239, June 1996.

${ }^{13}$ Moskowitz, M. E., Hrovat, K., Finkelstein, R., and Reckart, T., "SAMS Acceleration Measurements on MIR From September 1996 to January 1997," NASA TM-97-206320, Dec. 1997.

${ }^{14}$ DeLombard, R., "SAMS Acceleration Measurements on Mir from January to May 1997 (NASA Increment 4)," NASA TM-1998-208646, Oct. 1998.

${ }^{15}$ Neogi, N. A., and Newman, D. J., "Estimation of the Transfer Function for the Russian Space Station Mir due to Astronaut Loads," AIAA Paper 2000-1736, April 2000.

${ }^{16}$ Roberson, R. E., "Comments on the Incorporation of Man into the Attitude Dynamics of Spacecraft," Journal of the Astronautical Sciences, Vol. 10, No. 1, 1963, pp. 27, 28.

${ }^{17}$ DeLombard, R., "Compendium of Information for Interpreting the Microgravity Environment of the Orbiter Spacecraft," NASA TM-107032, Aug. 1996.

${ }^{18}$ Tryfonidis, M., "Robust Adaptive Control Modeling of Human Arm Movements Subject to Altered Gravity and Mechanical Loads," Ph.D. Dissertation, Dept. of Aeronautics and Astronautics, Massachusetts Inst. of Technology, Cambridge, MA, June 1999.

${ }^{19}$ Amir, A. R., "Design and Development of Advanced Load Sensors for the International Space Station," E.A.A. Thesis, Dept. of Aeronautics and Astronautics, Massachusetts Inst. of Technology, Cambridge, MA, Sept. 1998.

${ }^{20}$ Kim, H. M., and Bokhour, E. B., "Mir Structural Dynamics Experiment-A Flight Experiment Development," Proceeding s of the 38th AIAA/ASME/ASCE/AHS/ASC Structures, Structural Dynamics, and Materials Conference and Exhibit, and AIAA/ASME/AHS Adaptive Structures Forum, Collection of Technical Papers, Pt. 1, AIAA, Reston, VA, 1997, pp. $577-585$.

${ }^{21}$ Rabiner, L. R., and Gold, B., Theory and Application of Digital Signal Processing, Prentice-Hall, Englewood Cliffs, NJ, 1975. 